\title{
A collaborative pipeline
}

\author{
A meeting of the academic and industry minds may help fuel the development of a new generation of CNS drugs.
}

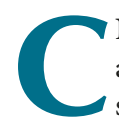
NS drug discovery is a vexing enterprise. The cost of developing a new drug can easily exceed a billion dollars, and the odds of success are slim. Despite major investments of capital, gains have been limited. Consider Alzheimer's disease, for instance, with an estimated clinical trial failure rate of over $99 \%$ and not a single diseasemodifying drug on the market ${ }^{1}$. From a public health perspective, the problem is large and growing. According to the World Health Organization, neurological disorders affect up to one billion people worldwide. Their prevalence is increasing, and the financial costs are mounting. Against this backdrop of urgent need, the recent decline in research and development investment by the pharmaceutical industry is a serious concern. As this issue of Nature Neuroscience went to press, GlaxoSmithKline announced that it was axing 900 jobs from its research workforce. While budget cuts have affected all disease portfolios, neuroscience has been among the hardest hit. This retreat raises the stark possibility that the CNS drug pipeline will dry up. It is time to consider new approaches, and academia and industry each have something to offer the drug discovery process. Bridging the cultural gap between these two communities may provide new ways to tackle this problem.

As governmental funding for translational projects has been on the rise, many basic neuroscientists have jumped on the translational bandwagon. More academic research focused on early-stage preclinical research is surely good news, but we must consider how to extend the reach of these efforts. There is a cultural gap to consider. In the 'publish or perish' environment, academics must focus on securing publications, and although they have considerable freedom in choosing what questions to pursue, funding cycles can restrict a study's scope and duration. To the average academic scientist, the resources of pharma may seem unlimited. Pharma is indeed in the privileged position of having the capital and infrastructure to bring a drug from bench to bedside, but industry scientists have their own concerns. With costs ballooning over the course of a drug's development, it is too risky to bring a drug to clinical trial unless strong evidence supports its ability to treat disease in humans. In this context, reproducibility issues that have plagued successful translation of basic research are a major obstacle to efficient drug discovery ${ }^{2}$. If academia is going to play a significant role in drug development, these issues must be addressed head on. Journals can contribute by enhancing reporting standards and encouraging data sharing, an ongoing effort at Nature Neuroscience. But the community must also agree on standards for disease research, with input from government and industry experts.

Understanding disease mechanisms is only one step on the long road to finding new treatments. A key step in translation is making the leap from preclinical models to humans. Most academic labs, however, do not have the expertise or the resources to develop the drugs that are essential for this translational milestone. For example, high-throughput assays and medicinal chemistry, rare in the average academic neurobiology lab, are critical for small molecule drug development. How, then, can the academic scientist contribute to the drug discovery process?

Fortunately, programs are being developed to stimulate such an integrated translational approach. For example, the US National Institutes of Health's Blueprint Neurotherapeutics Network (BPN) funds drug discovery ventures by academics and small biotech companies, supporting the small molecule discovery process from the initial chemistry steps up to phase 1 clinical trials. However, with a budget of $\sim$ \$60 million over its first five years of existence, the BPN can support only a modest number of projects. And to preserve the potential to attract investors, the projects funded by this initiative operate under secrecy, which can present a conundrum for academic researchers, as restrictions on publication can limit career advancement. New ways to recognize and reward academics who choose to invest their efforts in drug discovery must be developed and taken into account by academic institutions and funding agencies.

Universities have also stepped into the drug discovery arena with the establishment of academic drug discovery centers (ADDCs), which seek to recreate the drug discovery processes of big pharma on a smaller scale. Indeed, the rise of ADDCs has been associated with reverse migration of industry researchers to academia. This influx of scientists with industry experience can help provide key expertise for driving translational efforts in the academic setting.

Initiatives like the $\mathrm{BPN}$ and $\mathrm{ADDCs}$ are still relatively recent experiments, and it is too early to say how successful they will be in driving development of new medicines. But given the great unmet need for CNS therapies, it is encouraging to see the development of new ways to stimulate drug discovery, and these efforts should be fostered by universities and government agencies.

Academics should see these drug discovery ventures as an integral part of truly translational research and an opportunity to bring their work beyond the pages of journals and the screens of conferences, and to possibly benefit patients' lives. But what's in it for industry? The more advanced a project is along the axis of translation, the more likely it is to find investors to launch it into the clinical sphere. More integration of the drug discovery process and the industrial culture in academia means more value and less risk for the pharmaceutical industry. These are all good reasons for big pharma to consider re-investing in brain disease portfolios and increasing its financial stake in academic research. The first companies to re-invest will also be the first to reap the financial benefits of this concerted effort. Ultimately, joining forces may be the only way to achieve the breakthroughs so desperately needed for neurological disorders. By merging distinct perspectives and expertise, CNS drug discovery may become a winning proposition for everyone.

1. Cumming, J.L., Morstorf, T. \& Zhong, K. Alzheimers Res. Ther. 6, 37 (2014).

2. Prinz, F., Schlange, T. \& Asadullah, K. Nat. Rev. Drug Discov. 10, 712 (2011). 